\title{
Effects of moderate- and high-intensity aerobic training program in ambulatory subjects with incomplete spinal cord injury-a randomized controlled trial
}

\author{
Matthijs F. Wouda ${ }^{1} \cdot$ Eivind Lundgaard ${ }^{1} \cdot$ Frank Becker $^{1,2} \cdot$ Vegard Strøm $^{1}$
}

Received: 18 December 2017 / Revised: 5 April 2018 / Accepted: 16 April 2018 / Published online: 23 May 2018

(c) International Spinal Cord Society 2018

\begin{abstract}
Study Design Randomized controlled trial.

Objectives To investigate if high-intensity interval training (HIIT) exhibits a higher increase in physical capacity and activity levels compared to moderate-intensity training (MIT) and treatment as usual.

Setting Sunnaas Rehabilitation Hospital.

Methods Thirty ambulatory participants with incomplete spinal cord injury (SCI) were recruited at discharge from inpatient rehabilitation. Two intervention groups performed a 12-week individual training program at home by walking or running, depending on their physical ability. The MIT group was instructed to exercise three times a week at $70 \%$ of maximal heart rate (HRmax), while the HIIT group was instructed to exercise twice a week at 85-95\% of HRmax. The control group received treatment as usual. Pre- and post-tests consisted of maximal exercise testing on a treadmill (peak oxygen uptake (peak $\left.\mathrm{VO}_{2}\right)$ ), a 6-min walking test (6MWT), and 7 days of continuously activity monitoring (total daily energy expenditure (TDEE) and daily number of steps).

Results The HIIT, MIT, and control groups showed an increase in peak $\mathrm{VO}_{2}$ from pre- to post-test. However, no betweengroup difference in physical capacity (peak $\mathrm{VO}_{2}$ and 6MWT) and physical activity levels (TDEE and daily number of steps) were found between these groups.

Conclusions Performing HIIT did not exhibit a greater increase in physical capacity and activity levels than performing MIT or "treatment as usual" in ambulatory participants with SCI. Further studies are needed to elucidate both short- and long-term effects of HIIT and MIT in this SCI subpopulation.
\end{abstract}

\section{Introduction}

A spinal cord injury (SCI) can result in paralysis, sensory loss, and autonomic dysfunction. Depending on the injury level and the severity of damage along the spinal cord, clinical outcomes after a SCI can vary widely. In general, persons with chronic SCI tend to have low levels of

Electronic supplementary material The online version of this article (https://doi.org/10.1038/s41393-018-0140-9) contains supplementary material, which is available to authorized users.

Matthijs F. Wouda

Matthijs.wouda@sunnaas.no

1 Department of Research, Sunnaas Rehabilitation Hospital, Oslo, Norway

2 Faculty of Medicine, University of Oslo, Oslo, Norway physical activity [1]. Physical deconditioning can further exacerbate the impact of the injury and lead to an increased risk for chronic secondary health complications [2]. Although physical activity levels are found to increase during inpatient rehabilitation, it seems to decline after discharge [3]. Physical activity levels seem to play an important role in the fitness and health of persons with a SCI [4].

Low levels of physical capacity in the SCI population demand the promotion of physical activity to improve health and physical fitness in this population. Guidelines to improve physical fitness in those with SCI are developed [2] and later revised [5], and state that for cardiorespiratory fitness, adults with a SCI should engage in at least $20 \mathrm{~min}$ of moderate to vigorous intensity aerobic exercise 2 times per week [5]. The guidelines do not however differentiate injury levels or levels of completeness. Following these guidelines seems to have a positive effect on physical fitness in people 


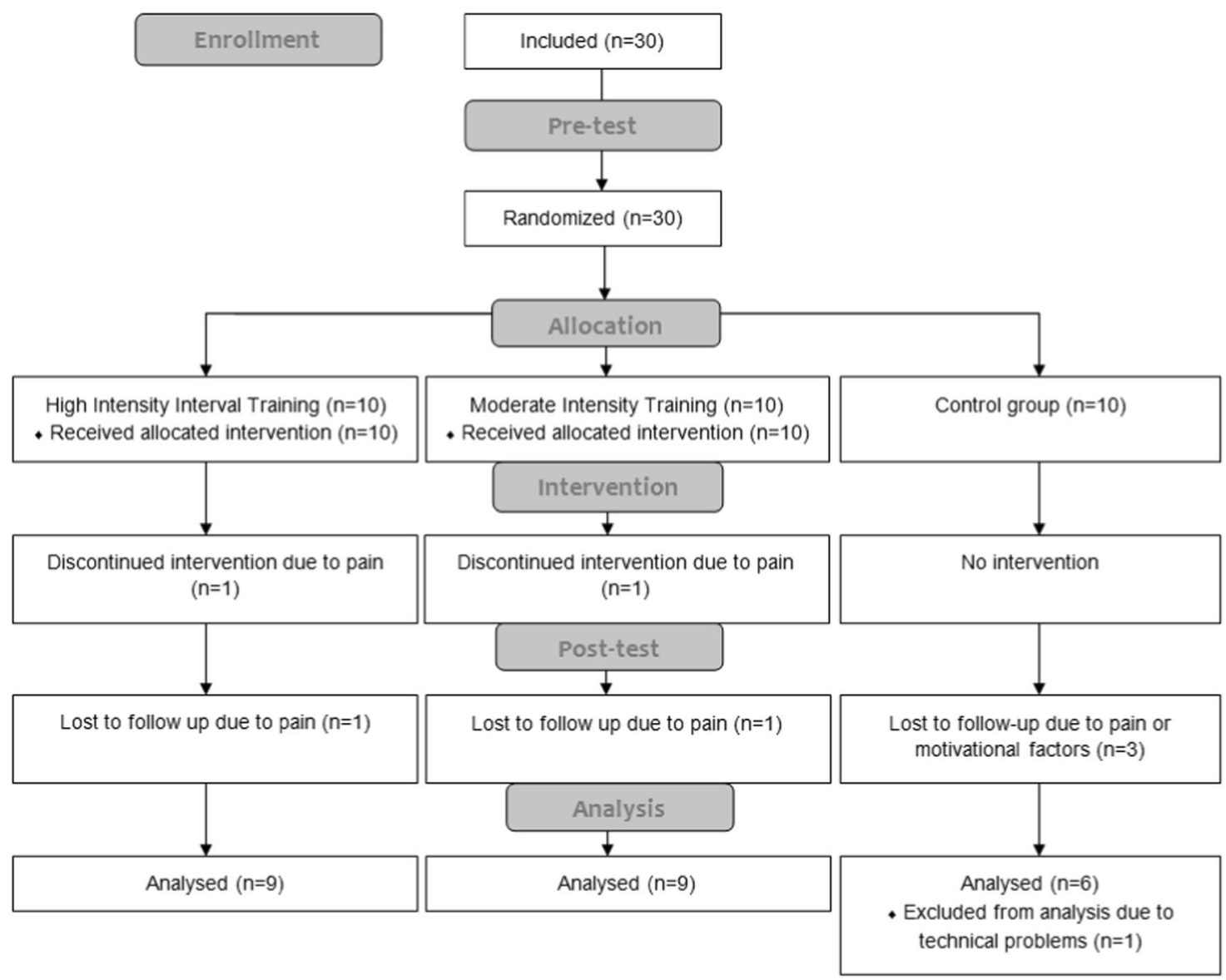

Fig. 1 Depiction of the study design and participants "flow" through the study

with SCI who are wheelchair dependent [6], but it is uncertain if the same positive effect is present in those with an incomplete SCI and the ability to walk.

The effects of aerobic exercise are highly dependent on the intensity with which the exercise is performed [7]. Aerobic exercise at high intensity, i.e., $85-95 \%$ of the maximal heart rate, and by engaging many large muscle groups seem to be most beneficial for increasing physical capacity [8]. Many persons with incomplete SCI, classified as AIS-D (according to the American Spinal Injury Association (ASIA) Impairment Scale (AIS)), are capable of performing high-intensity weight-bearing exercise, such as uphill walking $[9,10]$. High-intensity locomotor exercise in these subjects has also been shown to increase peak gait speed and improve gait pattern [11]. Increasing exercise intensity could offer a time-efficient solution to improve cardiometabolic health in those with SCI [12]. Highintensity aerobic exercise can be performed through repeated bouts of relatively intense exercise interspersed by short periods of recovery [13], e.g., high-intensity interval training (HIIT) [8]. HIIT programs by running and uphill walking are found to be very effective for increasing aerobic capacity in the able-bodied population $[8,13]$, as well as in persons with stroke or cardiometabolic diseases [14-16]. However, little is known about the impact of HIIT programs by walking/running on the physical fitness for persons with incomplete SCI. Therefore, studies examining the efficacy and feasibility of HIIT in this population are needed [12, 17].

The main objective of this study was to investigate the effects of a 12-week moderate- or high-intensity gait exercise intervention on physical capacity (peak oxygen uptake (peak $\left.\mathrm{VO}_{2}\right)$ ) and physical activity level (total daily energy expenditure (TDEE) and the daily number of steps) in ambulatory participants with SCI, soon after discharge from inpatient rehabilitation. We hypothesize that performing HIIT will exhibit a greater increase in physical capacity and activity levels compared to performing moderate-intensity training (MIT) or treatment as usual.

\section{Methods}

\section{Design}

Randomized controlled trial with three parallel groups. 


\section{Participants}

Thirty participants ( 25 men and 5 women) were recruited by consecutive enrollment over a 4-year period (2013-2017) during the last 2 weeks of inpatient rehabilitation at the hospital. In Norway, the average length of stay in hospital from acute care until the end of the inpatient rehabilitation was 121 days for traumatic SCI in 2014 [18]. The recruitment lasted 1 year after the scheduled date of closure. Although the intended sample size $(n=45)$ was not accomplished, recruitment had to be ended due to time constraints. Inclusion criteria were as follows: eligible persons were those aged between 18 and 70 years, with traumatic or non-traumatic incomplete (AIS-D) [19] SCI of all lesion levels, and being in their final phase of the subacute inpatient rehabilitation program at our hospital, i.e., about 2 weeks before discharge. There were no restrictions for inclusion with regard to wheelchair use, but they had to be able to walk on a treadmill for $5 \mathrm{~min}$ at $3 \mathrm{~km} / \mathrm{h}$ (without assistive walking aids). Participants were excluded if they had significant concurrent medical conditions that might limit their physical capacity, e.g., psychiatric conditions, orthopedic diseases, or uncontrolled cardiopulmonary disease.

\section{Pretest}

After medical approval for inclusion, the pretest was performed during the last week of the inpatient rehabilitation, comprising a maximal treadmill exercise test, a 6-min walking test (6MWT), and completion of the International Physical Activity Questionnaire (IPAQ). After completing the pretest, participants were randomized into one of the three groups (Fig. 1): HIIT group, MIT group, or a control group (treatment as usual). To achieve balance across the groups, block randomization was used, with a block size of 9 and an allocation ratio of 1:1:1. Lots were drawn from concealed envelopes by someone not involved in the test procedures. Outcome assessments were performed by two experienced staff members blinded to group allocation. The first week after discharge, physical activity level was monitored by wearing a portable activity monitor, Sensewear Armband (SWA), for seven consecutive days in their private home situation.

\section{Interventions}

Following the week with activity monitoring, participants in the two intervention groups performed a 12-week individual training program. Training intensity was based on the peak heart rate (peak HR), which was measured during maximal exercise testing (pretest). To ensure that participants in the intervention groups exercised at the predetermined intensity level, they received a heart rate monitor. They were taught how to use it during a moderate- or high-intensity training session. To assess intervention adherence, all participants (including those in the control group) were instructed to record all trainings sessions and other planned physical activities in a log. All participants received contact information to the research team in case they had any questions.

The HIIT program consisted of two sessions per week each lasting for $35 \mathrm{~min} ; 10 \mathrm{~min}$ warm-up at $70 \%$ of peak HR followed by $4 \times 4 \mathrm{~min}$ intervals at an intensity of $85-95 \%$ of peak HR interspersed with $3 \times 3$ min recovery periods at an intensity of $70 \%$ of peak HR [8].

The MIT program consisted of three sessions per week each lasting for $45 \mathrm{~min}$ of continuously activity at an intensity of $70 \%$ of peak HR.

For both training programs the weekdays to perform the training sessions were self-selected; however, they were requested to have at least 1 day in between the sessions. The training mode was either walking or running depending on the participants' physical capacity level and physical constraints. They were free to use a treadmill or to do the training outdoors. No cool-down was scheduled in any of the training programs.

The control group received "treatment as usual". In Norway, the specialized SCI rehabilitation units have a commitment to life-long follow-up for persons with SCI. After discharge from the first rehabilitation period, they are admitted for regular medical check-ups depending on their needs. In addition, they can contact the hospitals if they have specific issues that need solving. Most persons with SCI have regular contact with health care providers in the community, as a physiotherapist. Those in the control group did not receive any aerobic exercise prescriptions, but they had no restrictions with regard to physical training. They were only contacted once (by telephone) to schedule an appointment for the posttest.

\section{Posttest}

At 13 weeks after discharge, participants returned to the hospital for the posttests, repeating the same procedures as for the pretest. Participants were instructed not to communicate the type of intervention to staff members. Physical activity was again monitored during the 7 days after these tests (i.e., week 14 after discharge).

\section{Outcome measures}

Physical capacity was determined by measuring peak $\mathrm{VO}_{2}$ (pre-specified primary outcome measure), using a computerized standard open-circuit technique breath-by-breath spirometer (Vmax 220, Sensormedics Corporation, Yorba Linda, CA, USA) during a maximal graded exercise test on 
Table 1 Demographics and injury-specific characteristics of the participants

\begin{tabular}{|c|c|c|c|c|}
\hline & $\begin{array}{l}\text { HIIT }(n \\
=10)\end{array}$ & $\begin{array}{l}\text { MIT }(n \\
=10)\end{array}$ & $\begin{array}{l}\text { Control }(n \\
=10)\end{array}$ & $\begin{array}{l}\text { Total }(n \\
=30)\end{array}$ \\
\hline \multicolumn{5}{|l|}{ Demographics } \\
\hline $\begin{array}{l}\text { Age (years; mean } \\
(\mathrm{SD}))\end{array}$ & $50(15)$ & $34(15)$ & $41(19)$ & $41(17)$ \\
\hline $\begin{array}{l}\text { Body weight }(\mathrm{kg} \text {; } \\
\text { mean }(\mathrm{SD}))\end{array}$ & $86(20)$ & $77(15)$ & $80(13)$ & $81(16)$ \\
\hline $\begin{array}{l}\text { BMI }\left(\mathrm{kg} / \mathrm{m}^{2} ; \text { mean }\right. \\
(\mathrm{SD}))\end{array}$ & $\begin{array}{l}26.0 \\
(4.1)\end{array}$ & $\begin{array}{l}24.8 \\
(4.5)\end{array}$ & $25.5(4.5)$ & $25.4(4.2)$ \\
\hline Smoking $(n)$ & 1 & 3 & 1 & 5 \\
\hline Male/Female (ratio) & $8: 2$ & $8: 2$ & $9: 1$ & $25: 5$ \\
\hline \multicolumn{5}{|c|}{ Injury-specific characteristics } \\
\hline $\begin{array}{l}\text { Traumatic/non- } \\
\text { traumatic (ratio) }\end{array}$ & $9: 1$ & $6: 4$ & $9: 1$ & $24: 6$ \\
\hline $\begin{array}{l}\text { Time since injury } \\
\text { (days; mean (SD)) }\end{array}$ & $68(31)$ & $65(34)$ & $73(24)$ & $69(29)$ \\
\hline \multicolumn{5}{|l|}{ Neurological levela $(n)$} \\
\hline Cervical $1-8$ & 7 & 4 & 7 & 18 \\
\hline Thoracic $1-5$ & 1 & 1 & 1 & 3 \\
\hline Thoracic 6-12 & 0 & 3 & 0 & 3 \\
\hline Lumbar 1-5 & 2 & 2 & 1 & 5 \\
\hline Sacral $1-5$ & 0 & 0 & $1^{\mathrm{b}}$ & 1 \\
\hline
\end{tabular}

HIIT high-intensity interval training, MIT moderate-intensity training, $B M I$ body mass index

${ }^{a}$ Neurological injury level (International Standards for Neurological Classification of Spinal Cord Injury)

${ }^{\mathrm{b}}$ Apart from this subject (ASIA-A, SCI at level S2), all subjects had an incomplete SCI, ASIA-D

a treadmill (Woodway PPS Med, Waukesha, WI, USA). Additionally, respiratory exchange ratio (RER), peak HR (Polar M400), and blood lactate [La-] (BIOSEN C-line, Sport, EFK diagnostics, Barleben, Germany) were measured to evaluate whether criteria for maximal exercise testing were achieved: RER $(>1.15)$, peak HR $>85 \%$ of expected (for men: $>220-0.88 \times$ age, for females: 220 $-0.66 \times$ age $)$ and $[\mathrm{La}-](>8.0 \mathrm{mmol} / \mathrm{L})[20]$. The Sunnaas protocol for maximal exercise testing on a treadmill was used [9]. Speed and/or inclination increased gradually each minute until volitional fatigue was reached.

Participants performed the 6MWT (secondary outcome measure) to evaluate their walking ability. The number of meters walked and the heart rate after the test $\left(\mathrm{HR}_{\text {after-test }}\right)$, measured with a heart rate monitor (Polar M400, Kempele, Finland), were registered.

Physical activity levels were measured with the SenseWear ${ }^{\mathrm{TM}} \mathrm{PrO}_{2}$ Armband (SWA) (Bodymedia Inc., Pittsburgh, PA, USA), a small activity monitor attached on the right triceps. The TDEE (kilojoule (kJ) per min) and the daily number of steps were measured by SWA. To assess
TDEE into kilo calories (kcal) per min, the TDEE was divided by 4.1858 [21]. SenseWear Professional 7.0 (software) was used to analyze the data.

The IPAQ [22] was used to quantify the self-reported time spent on high-, moderate-, or low-intensity physical activity. The questionnaire was modified to quantify physical activity over a 3-month period, instead of 7 days. At pretest, the participants filled out the IPAQ recalling the 3 months before the time of injury and at posttest the preceding 3 months.

\section{Data analyses}

Assuming that those with an incomplete SCI show a similar training response as healthy participants [8], a sample size of 15 in each group would be sufficient to detect a clinically significant difference of $0.35 \mathrm{~L} / \mathrm{min}$ in peak $\mathrm{VO}_{2}$ between the HIIT group and control group. Sample size calculation was based on an assumption of a standard deviation of $0.4 \mathrm{~L} / \mathrm{min}$, a power of $80 \%$, and a significance level of $5 \%$.

Statistical analyses were performed with the Statistical Package for the Social Sciences (release 23.0.0.2 SPSS Inc., Chicago, IL, USA). Changes (\%) in physical capacity and physical activity levels from pre- to post-test were calculated group wise, based on the results from participants who had performed both pre- and post-test.

To determine whether peak $\mathrm{VO}_{2}, 6 \mathrm{MWT}$, TDEE, and daily number of steps changed differently in the HIIT, MIT, and control groups, a one-way analysis of covariance (ANCOVA) was conducted with pretest values as the covariate. ANCOVA can be used in a randomized pretest-posttest design to reduce error variance [23]. Group was used as fixed factor and posttest values as depended variables. The assumptions for performing ANCOVA in this randomized controlled trail were checked and met. For all tests, statistical significance was set at an alpha level of 0.05 .

\section{Results}

The baseline descriptive characteristics of the participants are presented in Table 1 . There were no statistically significant differences in the demographics or injury-specific characteristics between the groups.

On average, the mean body weight increased from pretest to posttest by $1 \%( \pm 3 \%), 1 \%( \pm 3 \%)$, and $3 \%( \pm 3 \%)$ in the HIIT, MIT and control groups, respectively.

The pre- and post-test results for physical capacity (peak $\mathrm{VO}_{2}$ and 6MWT) and physical activity levels (TDEE and daily amount of steps) are presented in Table 2 . 
Table 2 Pre- and post-test results for participants in all groups, mean (SD)

\begin{tabular}{|c|c|c|c|c|c|c|}
\hline \multirow[t]{2}{*}{ PHYSICAL CAPACITY } & \multicolumn{2}{|l|}{ HIIT } & \multicolumn{2}{|l|}{ MIT } & \multicolumn{2}{|l|}{ Control } \\
\hline & Pretest & Posttest & Pretest & Posttest & Pretest & Posttest \\
\hline $\begin{array}{l}\text { Graded maximal exercise } \\
\text { test }\end{array}$ & $(n=10)$ & $(n=9)$ & $(n=10)$ & $(n=9)$ & $(n=9)$ & $(n=7)$ \\
\hline Peak $\mathrm{VO}_{2}(\mathrm{~L} / \mathrm{min})$ & $2.70(0.81)$ & $3.00(0.62)$ & $2.79(0.79)$ & $3.23(0.94)$ & $2.78(0.57)$ & $3.15(0.59)$ \\
\hline Peak $\mathrm{VO}_{2}(\mathrm{ml} / \mathrm{kg} / \mathrm{min})$ & $32.1(9.1)$ & $35.7(5.3)$ & $36.9(11.8)$ & $42.3(12)$ & $35.6(6.0)$ & $40.7(6.5)$ \\
\hline Peak HR (beats/min) & $167(13)$ & $173(15)$ & $177(24)$ & $184(15)$ & $182(11)$ & $183(14)$ \\
\hline RER (ratio) & $1.23(0.10)$ & $1.21(0.71)$ & $1.17(0.14)$ & $1.19(0.07)$ & $1.24(0.06)$ & $1.22(0.06)$ \\
\hline$[\mathrm{La}-](\mathrm{mmol} / \mathrm{L})$ & $8.96(2.25)$ & $8.7(1.2)$ & $8.99(3.72)$ & $10.4(2.8)$ & $8.78(2.46)$ & $8.02(2.44)$ \\
\hline $6 \mathrm{MWT}$ & $(n=10)$ & $(n=9)$ & $(n=9)$ & $(n=9)$ & $(n=10)$ & $(n=7)$ \\
\hline Distance (m) & $561(93)$ & $654(96)$ & $573(96)$ & $678(136)$ & $607(77)$ & $638(80)$ \\
\hline $\mathrm{HR}_{\text {after-test }}($ beats/min$)$ & $118(17)$ & $134(21)$ & $145(29)$ & $152(32)$ & $143(21)$ & $142(24)$ \\
\hline \multicolumn{7}{|l|}{ PHYSICAL ACTIVITY } \\
\hline Activity monitoring & $(n=10)$ & $(n=9)$ & $(n=8)$ & $(n=7)$ & $(n=10)$ & $(n=6)$ \\
\hline TDEE (Kcal) & $2666(528)$ & 2744 (359) & 2736 (603) & 2768 (531) & 2437 (341) & $2697(405)$ \\
\hline Daily steps & $\begin{array}{l}5223 \\
(2737)\end{array}$ & $\begin{array}{l}6217 \\
(3080)\end{array}$ & $\begin{array}{l}6538 \\
(3580)\end{array}$ & $\begin{array}{l}6125 \\
(2175)\end{array}$ & $\begin{array}{l}5477 \\
(1624)\end{array}$ & $5057(922)$ \\
\hline
\end{tabular}

HIIT high-intensity interval training, $M I T$ moderate-intensity training, $\mathrm{VO}_{2}$ oxygen uptake, $H R$ heart rate, $R E R$ respiratory exchange ratio, $[\mathrm{La}-]$ blood lactate, $6 M W T 6 \mathrm{~min}$ walking test, TDEE total daily energy expenditure

\section{Physical capacity}

The peak $\mathrm{VO}_{2}(\mathrm{~L} / \mathrm{min})$ increased from the pre- to the posttest on average $13 \%( \pm 17 \%), 8 \%( \pm 13 \%)$, and $10 \%( \pm 7 \%)$ in the HIIT, MIT and control groups, respectively. Similarly, the distance walked during the 6MWT increased on average by $18 \%( \pm 11 \%), 15 \%( \pm 16 \%)$, and $9 \%( \pm 15 \%)$, respectively.

The ANCOVA (Table 3), however, revealed no statistically significant differences in changes from the pre- to the post-test between the groups after controlling for the pretest values in either peak $\mathrm{VO}_{2}(\mathrm{~L} / \mathrm{min}), F(2,20)=0.06, p=$ 0.94 , or 6MWT (meters), $F(2,21)=0.55, p=0.58$ ).

\section{Physical activity levels}

TDEE increased from the pre- to post-test on average 7\% $( \pm 11 \%), 1 \%( \pm 15 \%)$, and $5 \%( \pm 10 \%)$ in the HIIT, MIT and control groups, respectively. The MIT and control group had a decreased daily number of steps on average $-1 \%$ $( \pm 54 \%)$ and $-1 \%( \pm 39 \%)$, while the HIIT group showed an average increase of $16 \%( \pm 18 \%)$. However, ANCOVA revealed no significant effect of group on the changes in the physical activity levels (see Table 3$)$ : TDEE $(F(2,17)=$ $0.24, p=0.79)$ and daily amount of steps $(F(2,17)=0.48$, $p=0.63$ ).

In all groups, large individual variations in changes from pre- to post-test were evident in peak $\mathrm{VO}_{2}$ (Fig. 2), daily number of steps (Fig. 3), and TDEE (Fig. 4).
Table 3 Between-group differences in estimated marginal means (with 95\% CI and $p$ value) of the changes from pre- to post-test in the physical capacity and physical activity outcome measures

\begin{tabular}{lllll}
\hline & Group & Difference $^{\mathrm{a}}$ & $95 \%$ CI & $p$ Value \\
\hline Peak $\mathrm{VO}_{2}(\mathrm{~L} / \mathrm{min})$ & HIIT $^{\mathrm{b}}$ & & & \\
& MIT & -0.04 & -0.45 to 0.38 & 0.86 \\
& Control & -0.08 & -0.53 to 0.38 & 0.73 \\
Peak $\mathrm{VO}_{2}(\mathrm{ml} / \mathrm{kg} /$ & HIIT $^{\mathrm{b}}$ & & & \\
min) & MIT & +1.0 & -3.9 to 6.0 & 0.66 \\
& Control & +0.3 & -5.1 to 5.6 & 0.91 \\
6MWT (m) & HIIT & & & \\
& MIT & -2 & -85 to 81 & 0.96 \\
& Control & -40 & -129 to 48 & 0.36 \\
TDEE (Kcal) & HIIT & & & \\
& MIT & -79 & -372 to 215 & 0.58 \\
& Control & -91 & -414 to 232 & 0.56 \\
Daily amount of & HIIT & & & \\
steps & MIT & -532 & -2518 to & 0.58 \\
& & & 1454 & \\
& Control & -985 & -3168 to & 0.35 \\
& & & 1198 & \\
\hline
\end{tabular}

95\% CI 95\% confidence interval, $V_{2}$ oxygen uptake, HIIT highintensity interval training, MIT moderate-intensity training, $6 M W T 6$ min walking test, TDEE total daily energy expenditure

${ }^{a}$ Differences in mean change from pre- to post-test between the HIIT group (reference) and the MIT and control group, while controlling for pretest values

${ }^{\mathrm{b}} \mathrm{HIIT}$ group is used as reference group 


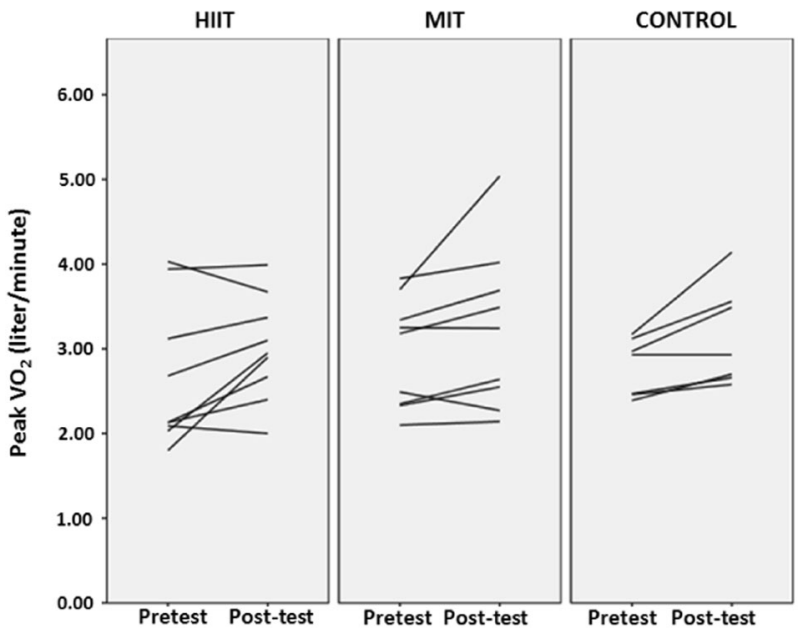

Fig. 2 Individual changes in peak $\mathrm{VO}_{2}(\mathrm{~L} / \mathrm{min})$ from pre- to post-test in HIIT, MIT, and control groups. HIIT high-intensity interval training, MIT moderate-intensity training, $\mathrm{VO}_{2}$ oxygen uptake

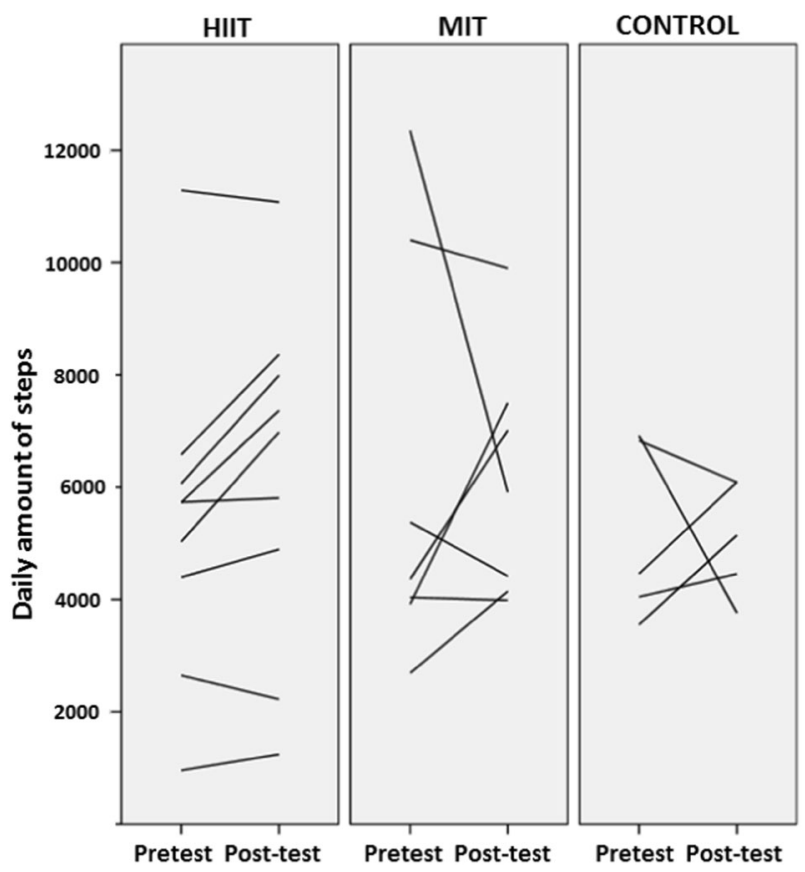

Fig. 3 Individual changes in daily number of steps from pre- to posttest in the HIIT, MIT, and control groups. HIIT high-intensity interval training, MIT moderate-intensity training

\section{Self-reported time spent on physical activity}

Table 4 shows the amount of time participant spent on physical activity at moderate and high intensity, selfreported by the IPAQ during the last 3 months before injury and during the intervention period.

\section{Attrition and adherence to the training program}

The HIIT group had one drop-out due to pain (not exercise induced), while the MIT group had one drop-out due to

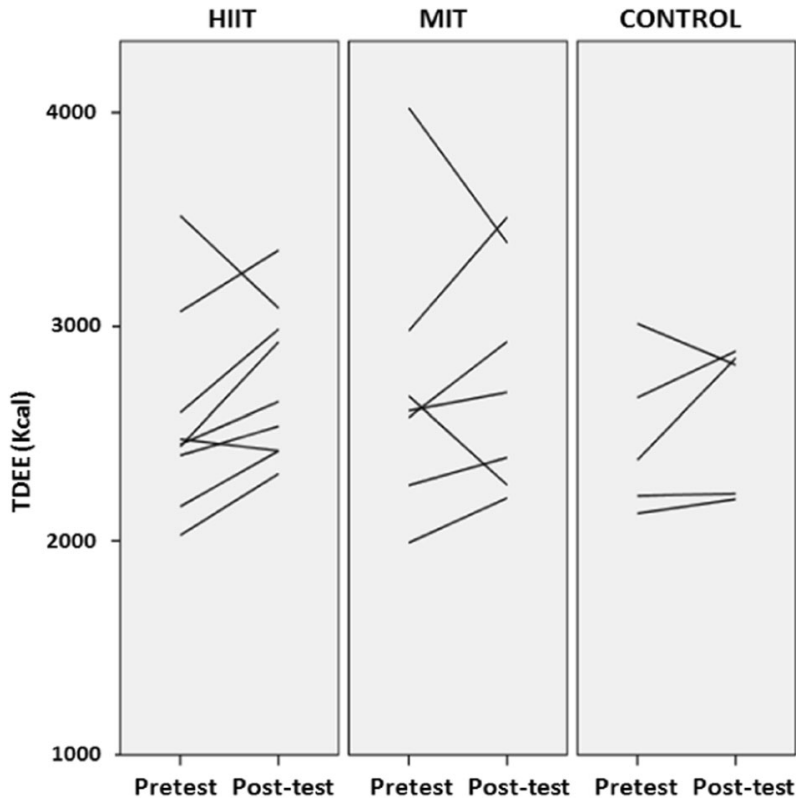

Fig. 4 Individual changes in TDEE from pre- to post-test in the HIIT, MIT, and control groups. HIIT high-intensity interval training, MIT moderate-intensity training, TDEE total daily energy expenditure

motivational factors. The control group had three drop-outs due to comorbidity and motivational factors and the results from one exercise test could not be used for analysis due to technical problems. No adverse events were reported during either the HIIT or MIT training.

Totally, 18 of 30 training logs were returned: HIIT: $n=$ 9, MIT: $n=4$ and control group: $n=5$.

The $\operatorname{logs}$ revealed that participants during the 12 -week intervention period had on average performed totally 49 (HIIT), 53 (MIT), and 55 (controls) training sessions.

\section{Discussion}

The main finding of the present study was that HIIT was not superior to MIT or "treatment as usual" in improving peak $\mathrm{VO}_{2}$ or physical activity measures following a 12-week training program in ambulatory participants with incomplete SCI. We hypothesized, however, that performing HIIT would exhibit a greater increase in physical capacity and activity levels, compared to moderate-intensity training. The participants of each group did, on average, elicit an elevated peak $\mathrm{VO}_{2}$ corresponding approximately one metabolic equivalent which equals $3.5 \mathrm{ml} / \mathrm{kg} / \mathrm{min}$. Such an increase could, from a clinical perspective, have a great impact on a person's physical capacity, and might also be associated with improvement in survival [24]. The improvement in peak $\mathrm{VO}_{2}$ found in the present study is similar to other studies on the effects of high-intensity exercise programs in healthy subjects $[8,25]$. In ambulatory 
Table 4 Weekly amount (median (IQR, min-max)) of time (min) spent on physical activity at moderate and high intensity in the HIIT, MIT and control groups during the last 3 months before injury and during the intervention period, self-reported by the IPAQ

\begin{tabular}{llllll}
\hline & \multicolumn{2}{l}{ During the 3 months before injury } & & \multicolumn{2}{l}{ During the intervention period } \\
\cline { 2 - 3 } \cline { 5 - 6 } & $\begin{array}{l}\text { High-intensity } \\
\text { physical activity }\end{array}$ & $\begin{array}{l}\text { Moderate intensity } \\
\text { physical exercise }\end{array}$ & & $\begin{array}{l}\text { High-intensity } \\
\text { physical activity }\end{array}$ & $\begin{array}{l}\text { Moderate-intensity } \\
\text { physical activity }\end{array}$ \\
\hline $\begin{array}{l}\text { HIIT group } \\
\text { MIT group }\end{array}$ & $68(74,0-240)$ & $60(180,0-480)$ & & $180(93,23-360)$ & $135(128,0-480)$ \\
$\begin{array}{l}\text { Control } \\
\text { group }\end{array}$ & $135(424,0-540)$ & $158(518,0-1350)$ & & $210(150,0-270)$ & $240(263,30-495)$ \\
\hline
\end{tabular}

$I Q R$ interquartile range, min minimum, max maximum, HIIT high-intensity interval training, MIT moderateintensity training, IPAQ International Physical Activity Questionnaire persons with incomplete SCI, DiPiro et al. [26] showed that a high-intensity exercise program, using a recumbent stepper, significantly increased peak $\mathrm{VO}_{2}$. Those in the MIT group exhibited similar increase in peak $\mathrm{VO}_{2}$ as for the HIIT group. This could be explained by the rather low baseline physical capacity levels of the participants, which is about $15 \%$ lower than reported in the healthy Norwegian population [27]. Individuals with low physical capacity seem to respond better on exercise at a lower intensity than those with an averagely physical capacity [7]. On the other hand, individuals with high levels of aerobic physical capacity do not seem to respond to aerobic training at moderate intensity [8].

Surprisingly, those in the control group also increased the peak $\mathrm{VO}_{2}$ from pre- to post-test, and similar to that in the intervention groups. Summed results from other studies [28] show on average $5.5 \mathrm{ml} / \mathrm{kg} / \mathrm{min}$ higher peak $\mathrm{VO}_{2}$ increase after high-intensity exercise vs. (none-exercising) control groups.

The minimal clinically important difference for the 6MWT is estimated to be 54-80 m [29]. An increase of 99 and $90 \mathrm{~m}$, respectively, indicates that both HIIT and MIT interventions had a clinically significant effect on the walking ability of the participants in this study. However, in the HIIT group, the $\mathrm{HR}_{\text {after-test }}$ was considerably higher after the posttest than after the pretest. The increased number of meters walked during the 6MWT could be explained by the higher level of intensity this test was performed at.

In the recently updated guideline recommendations [5], persons with SCI are encouraged to do upper body aerobic exercise to improve cardiorespiratory fitness. Of the 30 participants in the present study, 27 achieved at least two of the established criteria for maximal exercise testing [20], confirming other studies that ambulatory persons with incomplete SCI are able to exercise at high intensity by ambulation [9-11]. Furthermore, our study indicates that for ambulatory persons with SCI both HIIT and MIT training programs by weight-bearing exercises as uphill walking are feasible and might be favorable in order to improve physical capacity.

The activity monitoring revealed that the HIIT group at 3 months after admission from the rehabilitation hospital on average had increased the number of steps per day by 836 . Despite this increase, the number of steps a day did, however, not differ significantly from the other groups. Also, it is uncertain if such an increase is sufficient to gain positive health effects [30]. Physical activity is positively associated with several health outcomes in healthy persons as in those with SCI [31, 32]. Thus, HIIT could eventually lead to an improved cardiometabolic health in persons with incomplete SCI. The activity monitoring also revealed that most participants in this study had rather low levels of physical activity compared to healthy persons [33].

There are some limitations to the current study that warrant discussion. First, the intended sample size $(n=45)$ was not accomplished as 15 fewer subjects than expected were included even after 1-year elongation of the planned recruitment period. The reasons for this might be ascribed to not only commitment challenges due to motivational aspects and pain, but also because several potential participants had to be excluded due to comorbidity. The present participants are thus assumed to be highly motivated for doing physical activity, as might also be reflected by the high drop-out rate in the control group. Secondly, an unexpected finding was that those in the control group, left to treatment as usual, exhibited similar responses at the posttest on both physical capacity and physical activity measures as those in the intervention groups. Due to ethical considerations, participants in this group had no restrictions regarding training volume or intensity. Participants in the control group did not seem to exercise less at high intensity compared to those in the intervention groups during the intervention period (Table 4). Probably, several participants in the control group considered high-intensity exercise as part of the "treatment as usual", and thus we were in the present study left without a "non-exercising" control group. Thirdly, adherence is an important factor contributing to the effectiveness of exercise-based rehabilitation [34]. Unfortunately, there is to our knowledge no cheap and easily available gold standard measurement of unsupervised exercise-based rehabilitation adherence available [34]. In our study we designed our own training logs. Only 4 and 5 out of 10 training logs were returned in the MIT and control group, respectively, making it difficult to explain between- 
group differences. Therefore, participants' adherence to the training frequency and intensity in the intervention group could not be monitored sufficiently in this study. Participants in the MIT and HIIT groups used HR monitors to ensure accurate training intensity. However, to evaluate adherence, HR data should have been collected over the whole intervention period.

In conclusion, performing a 12-week high-intensity interval training program, shortly after discharge from inpatient rehabilitation, did not exhibit a greater increase in physical capacity and activity levels than performing moderate-intensity training or "treatment as usual" in ambulatory participants with SCI. Both HIIT and MIT seem to be feasible in this SCI subpopulation. Further studies are, however, needed to elucidate the short- and long-term effects.

Author contributions MFW was responsible for designing and writing the research protocol, analyzing data, interpreting results and writing the report. EL contributed to designing the research protocol, testing of the participants and contributed to writing the report. FB contributed to designing the research protocol, interpreting results and provided feedback on the report. VS was responsible for designing and writing the research protocol, analyzing data, interpreting results and contributed to writing the report.

Funding This research was partly sponsored by the Birgit og Rolf Sunnaas Minnefond.

\section{Compliance with ethical standards}

Statement of ethics We certify that all applicable institutional and governmental regulations concerning the ethical use of human volunteers were followed during the course of this research.

The present study has been registered in ClinicalTrials.gov (Identifier: NCT01903226, https://clinicaltrials.gov/show/NCT01903226) and was approved by the Regional Medical Ethical Research Committee. All participants signed an informed consent after being informed about the purpose, procedures, and potential risks of the study by giving written and oral information.

Conflict of interest The authors declare that they have no conflict of interest.

\section{References}

1. Buchholz AC, McGillivray CF, Pencharz PB. Physical activity levels are low in free-living adults with chronic paraplegia. Obes Res. 2003;11:563-70.

2. Ginis KA, Hicks AL, Latimer AE, Warburton DE, Bourne C, Ditor DS, et al. The development of evidence-informed physical activity guidelines for adults with spinal cord injury. Spinal Cord. 2011;49:1088-96.

3. van den Berg-Emons RJ, Bussmann JB, Haisma JA, Sluis TA, van der Woude LH, Bergen MP, et al. A prospective study on physical activity levels after spinal cord injury during inpatient rehabilitation and the year after discharge. Arch Phys Med Rehabil. 2008;89:2094-101.

4. Nooijen CF, de Groot S, Postma K, Bergen MP, Stam HJ, Bussmann JB, et al. A more active lifestyle in persons with a recent spinal cord injury benefits physical fitness and health. Spinal Cord. 2012;50:320-3.

5. Martin Ginis KA,van der Scheer JW,Latimer-Cheung AE,Barrow A,Bourne C,Carruthers P, et al. Evidence-based scientific exercise guidelines for adults with spinal cord injury: an update and a new guideline. Spinal Cord. 2018;56:308-21.

6. Pelletier CA, Totosy de Zepetnek JO, MacDonald MJ, Hicks AL. A 16-week randomized controlled trial evaluating the physical activity guidelines for adults with spinal cord injury. Spinal Cord. 2015;53:363-7.

7. Swain DP, Franklin BA. VO(2) reserve and the minimal intensity for improving cardiorespiratory fitness. Med Sci Sports Exerc. 2002;34:152-7.

8. Helgerud J, Hoydal K, Wang E, Karlsen T, Berg P, Bjerkaas M, et al. Aerobic high-intensity intervals improve VO2max more than moderate training. Med Sci Sports Exerc. 2007;39:665-71.

9. Lundgaard E,Wouda MF, Strom V. A comparative study of two protocols for treadmill walking exercise testing in ambulating subjects with incomplete spinal cord injury. Spinal Cord. 2017;55:935-9.

10. Wouda MF, Wejden L, Lundgaard E, Strom V. Energetic and cardiovascular responses to treadmill walking and stationary cycling in subjects with incomplete spinal cord injury. Spinal Cord. 2016;54:51-6.

11. Leech KA, Kinnaird CR, Holleran CL, Kahn J, Hornby TG. Effects of locomotor exercise intensity on gait performance in individuals with incomplete spinal cord injury. Phys Ther. 2016;96:1919-29.

12. Nightingale TE,Metcalfe RS,Vollaard NB,Bilzon JL. Exercise guidelines to promote cardiometabolic health in spinal cord injured humans: time to raise the intensit? Arch Phys Med Rehabil. 2017;98:1693-1704.

13. MacInnis MJ,Gibala MJ. Physiological adaptations to interval training and the role of exercise intensity. J Physiol. 2017;595:2915-30.

14. Munari D, Pedrinolla A, Smania N, Picelli A, Gandolfi M, Saltuari L, et al. High-intensity treadmill training improves gait ability, VO2peak and cost of walking in stroke survivors: preliminary results of a pilot randomized controlled trial. Eur J Phys Rehabil Med. 2016. [Epub ahead of print 30 Aug 2016].

15. Gjellesvik TI, Brurok B, Hoff J, Torhaug T, Helgerud J. Effect of high aerobic intensity interval treadmill walking in people with chronic stroke: a pilot study with one year follow-up. Top Stroke Rehabil. 2012;19:353-60.

16. Weston KS, Wisloff U, Coombes JS. High-intensity interval training in patients with lifestyle-induced cardiometabolic disease: a systematic review and meta-analysis. $\mathrm{Br} \mathrm{J}$ Sports Med. 2014;48:1227-34.

17. Astorino TA,Thum JS. Within-session responses to high-intensity interval training in spinal cord injury. Disabil Rehabil. 2018;40:444-9.

18. Strom V, Manum G, Leiulfsrud A, Wedege P, Rekand T, Halvorsen A, et al. People with spinal cord injury in Norway. Am J Phys Med Rehabil. 2017;96:S99-S101.

19. Kirshblum SC, Burns SP, Biering-Sorensen F, Donovan W, Graves DE, Jha A, et al. International standards for neurological classification of spinal cord injury (revised 2011). J Spinal Cord Med. 2011;34:535-46.

20. Edvardsen E, Hem E, Anderssen SA. End criteria for reaching maximal oxygen uptake must be strict and adjusted to sex and age: a cross-sectional study. PLoS One. 2014;9:e85276.

21. Mcardle WD KF, Katch VL. Exercise physiology: energy, nutrition and human performance. 6th ed. Philadephia: Lippincott Williams \& Wilkins; 2007. 
22. Kurtze N, Rangul V, Hustvedt BE, Flanders WD. Reliability and validity of self-reported physical activity in the Nord-Trondelag Health Study: HUNT 1. Scand J Public Health. 2008;36:52-61.

23. Dimitrov DM, Rumrill PD Jr. Pretest-posttest designs and measurement of change. Work. 2003;20:159-65.

24. Myers J, Prakash M, Froelicher V, Do D, Partington S, Atwood JE. Exercise capacity and mortality among men referred for exercise testing. New Engl J Med. 2002;346:793-801.

25. Gormley SE, Swain DP, High R, Spina RJ, Dowling EA, Kotipalli US, et al. Effect of intensity of aerobic training on VO2max. Med Sci Sports Exerc. 2008;40:1336-43.

26. DiPiro ND, Embry AE, Fritz SL, Middleton A, Krause JS, Gregory CM. Effects of aerobic exercise training on fitness and walking-related outcomes in ambulatory individuals with chronic incomplete spinal cord injury. Spinal Cord. 2016;54:675-81.

27. Edvardsen E, Hansen BH, Holme IM, Dyrstad SM, Anderssen SA. Reference values for cardiorespiratory response and fitness on the treadmill in a 20- to 85-year-old population. Chest. 2013;144:241-8.

28. Milanovic Z, Sporis G, Weston M. Effectiveness of high-intensity interval training (HIT) and continuous endurance training for VO2max improvements: a systematic review and meta-analysis of controlled trials. Sports Med. 2015;45:1469-81.
29. Wise RA, Brown CD. Minimal clinically important differences in the six-minute walk test and the incremental shuttle walking test. COPD. 2005;2:125-9.

30. Tudor-Locke C, Craig CL, Aoyagi Y, Bell RC, Croteau KA, De Bourdeaudhuij I, et al. How many steps/day are enough? For older adults and special populations. Int $\mathrm{J}$ Behav Nutr Phys Act. 2011;8:80.

31. Myers J, Lee M, Kiratli J. Cardiovascular disease in spinal cord injury: an overview of prevalence, risk, evaluation, and management. Am J Phys Med Rehabil. 2007;86:142-52.

32. Wahman K, Nash MS, Lewis JE, Seiger A, Levi R. Increased cardiovascular disease risk in Swedish persons with paraplegia: The Stockholm spinal cord injury study. J Rehabil Med. 2010;42:489-92.

33. Tudor-Locke C, Bassett DR Jr. How many steps/day are enough? Preliminary pedometer indices for public health. Sports Med. 2004;34:1-8.

34. Bollen JC, Dean SG, Siegert RJ, Howe TE, Goodwin VA. A systematic review of measures of self-reported adherence to unsupervised home-based rehabilitation exercise programmes, and their psychometric properties. BMJ Open. 2014;4:e005044. 